\title{
Management of post-operative sore throat in patients undergoing endotracheal intubation: literature review
}

\author{
Ahmed M. El Sharkawy ${ }^{1,2 *}$, Mustafa M. Rawas ${ }^{3}$, Amany M. Altoies ${ }^{4}$, \\ Mohammed A. Al Arbash ${ }^{5}$, Abdulaziz A. Alzahrani ${ }^{6}$, Anas M. Alnahari ${ }^{7}$, Khaled S. Alomari ${ }^{8}$, \\ Ziyad M. Hammad ${ }^{9}$, Basel M. Ebrahim ${ }^{10}$, Mansour M. Alqahtani ${ }^{11}$, Ali S. Alshahrani ${ }^{12}$
}

\author{
${ }^{1}$ Department of Anaesthesia, Khulais General Hospital, Khulais, Saudi Arabia \\ ${ }^{2}$ Department of Anaesthesia and Intensive Care, Faculty of Medicine, El Azhar University, Cairo, Egypt \\ ${ }^{3}$ Department of Anaesthesia, East Jeddah Hospital, Jeddah, Saudi Arabia \\ ${ }^{4}$ College of Medicine, King Saud bin Abdulaziz University for Health Sciences, Riyadh, Saudi Arabia \\ ${ }^{5}$ Department of Emergency Medicine, Dammam Medical Complex, Dammam, Saudi Arabia \\ ${ }^{6}$ Department of Anaesthesia, Tomas Bata Regional Hospital, Zlin, Czech Republic \\ ${ }^{7}$ General Physician, Ministry of Health, Riyadh, Saudi Arabia \\ ${ }^{8}$ College of Medicine, Ibn Sina National College, Jeddah, Saudi Arabia \\ ${ }^{9}$ Intern, King Abdulaziz University Hospital, Jeddah, Saudi Arabia \\ ${ }^{10}$ Department of General Surgery, King Hamad University Hospital, Manama, Bahrain \\ ${ }^{11}$ Department of Anaesthesia, Taif University, Taif, Saudi Arabia \\ ${ }^{12}$ General Physician, Armed Forces Hospital Southern Region, Abha, Saudi Arabia
}

Received: 19 December 2020

Revised: 31 December 2020

Accepted: 04 January 2021

*Correspondence:

Dr. Ahmed M. El Sharkawy,

E-mail: Ahelsharkawy@moh.gov.sa

Copyright: (c) the author(s), publisher and licensee Medip Academy. This is an open-access article distributed under the terms of the Creative Commons Attribution Non-Commercial License, which permits unrestricted non-commercial use, distribution, and reproduction in any medium, provided the original work is properly cited.

\begin{abstract}
Postoperative sore throat (POST) is a common complaint that is classified as the 8th most undesirable events after general anesthesia. Endotracheal intubation has been estimated to be the commonest factor causing POST. We aim to review the risk factors and preventive measures to reduce the incidence and severity of POST following endotracheal intubation. Although sore throat can be induced by many perioperative factors, endotracheal intubation remains the commonest cause that can induce endothelial injury and a subsequent sore throat. Many risk factors for this association has been reported. The most common risk factors include ages less than 60 years of age, female sex, cough, and asthma, underlying chest diseases, type and timing of the operation, type, and size of the used endotracheal tube. Moreover, the experience of the personnel intubating the patients may have been another risk factor. Medical prevention of POST can be achieved by several agents as steroids, non-steroidal anti-inflammatory drugs (NSAIDS), N-methyl-D-aspartate (NMDA) receptor antagonists, careful monitoring of the tracheal cuff pressure, and licorice application. On the other hand, using lidocaine has been contraindicated according to previous meta-analysis results. Additionally, we recommend that future studies with bigger sample sizes are should be conducted for further validation of the new modalities.
\end{abstract}

Keywords: Sore throat, Endotracheal, Intubation, Treatment, Prevention, Risk factors 


\section{INTRODUCTION}

Postoperative sore throat (POST) is a common complaint that is classified as the $8^{\text {th }}$ most undesirable events after general anesthesia. ${ }^{1}$ The incidence for this event has been recorded to be ranging between $40 \%$ and $85.7 \% .^{2-4}$ Many factors have been identified to cause POST. These include manipulation of the airways during the surgery, and choosing an inappropriate airway device. ${ }^{4}$ However, it usually happens secondary to endotracheal intubation that may lead to the destruction of the pharyngeal mucosa and subsequent injury resulting from the endotracheal cuff pressure. ${ }^{5,6}$ Higgins et al reported estimated that POST incidence rates of $45.4 \%, 17.5 \%$, and $3.3 \%$ were associated with endotracheal intubation, laryngeal masks, and open masks, respectively. ${ }^{4}$

Furthermore, many factors have been reportedly associated with an increased incidence of POST after endotracheal intubation. Hu et al. ${ }^{7}$ showed that lower incidence rates were associated with using smaller endotracheal tubes. Other factors include a rough introduction of the tube, suction, post endotracheal intubation, and longer periods of anesthesia. ${ }^{8}$ Additionally, higher POST rates were also associated with factors like age below 65 years old, being female, smoking, head and neck operations, asthma, and dry cough. ${ }^{9,10}$

Stout $e$ al categorized the severity of POST on a scale of $0-4 .{ }^{11}$ The peak severity of POST has been recorded at two to four hours after the extubations, but then the severity gradually subsides in the following day. ${ }^{12}$

In addition to the annoying feeling that patients usually experience, POST occurrence is also associated with the unpleasant feeling regarding the recovery from anesthesia and the quality of it. Therefore, it is recommended to take precautions for the prevention of this annoying event. Various approaches including pharmacological and nonpharmacological procedures to relieve POST have been previously reported. These non-pharmacological approaches are mainly prophylactic steps that intervene against the occurrence of pharyngeal injuries. Such approaches include the application of cuffed tracheal tubes in addition to using supraglottic airway apparatuses, decreasing the intracuff pressure, gentle esophageal approaches, and removing the tube after the tracheal cuff has been fully deflated. ${ }^{8}$

On the other hand, the pharmacological interventions include the application of perioperative agents that can reduce inflammation and intervene against the development of POST. These include corticosteroids, nonsteroidal anti-inflammatory drugs (NSAIDs), ketamine, $\alpha_{2}$-agonists, and local anesthetic drugs. ${ }^{13-17}$

In this study, we aim to review the risk factors of POST and the reported treatment modalities that are usually used to decrease its incidence and severity.

\section{METHODS}

We performed an extensive literature search of the Medline, Cochrane, and EMBASE databases on 18 October 2020 using the medical subject headings (MeSH) "sore throat [MeSH Terms]". Papers discussing the risk factors of POST, its prvention, or treatment modalities; were screened for relevant information. There were no limits on date, language, and age of participants or publication type.

\section{DISCUSSION}

\section{Risk factors for POST after endotracheal intubation}

Although sore throat can be induced by many perioperative factors, endotracheal intubation remains the commonest cause that can induce endothelial injury and a subsequent sore throat. Many risk factors for this association has been reported.

\section{Association between POST and patient-related factors}

Lee et al conducted a prospective study in Korean patients and included 207 patients to study the incidence and risk factors of POST following endotracheal intubation. ${ }^{18}$ The authors found that, by multivariate analysis, high intracuff pressure $\left(\geq 17 \mathrm{~cm} \mathrm{H}_{2} \mathrm{O}\right)$ and cough were the only risk factors for developing POST after endotracheal intubation. Another prospective study with a bigger sample size $(\mathrm{n}=809)$, conducted by Biro et al found that being female, longer durations of anesthesia, the presence of underlying lung disease, and the presence of blood on the tube on extubation were the most probable risk factors for developing POST following tracheal intubation. ${ }^{2}$ On the other hand, age was reportedly inversely correlated with developing POST. ${ }^{19}$ Additionally, Jaensson et al conducted a cross-sectional study and included female patients only to find the risk factors for developing a sore throat. ${ }^{20}$ The authors found that, among eight different factors that were analyzed, old age $(>60)$, using a throat pack, and the size of the used endotracheal tube (size $\geq 7$ $\mathrm{mm}$ ) were the only possible associated risk factors. Moreover, sex remains a controversial point. Although it has been reported that being female will most likely increase the incidence of OIST, it can be justified that women tend to not report their postoperative complications which may have biased the results. ${ }^{21}$ Jaensson $e t$ al also conducted a cross-sectional study to find the possible risk factors for each gender. ${ }^{22}$ For males, the main risk factor was being intubated by medical care personnel with $<3$ months of experience.

However, Inoue et al results showed that no significant difference was estimated in the incidence of POST between trainees and consultants in a big population study. ${ }^{19}$ On the other hand, multiple laryngoscopies and tube size (size $\geq 7 \mathrm{~mm}$ ) were the commonest risk factors among female patients. ${ }^{22}$ 


\section{POST and the size of the endotracheal tube}

Indeed, factors related to the trachea used in the intubation process are more likely to be more common than factors related to the patient's demographics. Jaensson et al conducted a meta-analysis in 2009 to investigate the association between the endotracheal tube size and incidence of sore throat in 100 female patients. ${ }^{12}$ The authors reported that a larger tube (size $\geq 7 \mathrm{~mm}$ ) was significantly more associated with a higher incidence of the sore throat than smaller endotracheal tubes ( size $\leq 6$ $\mathrm{mm})$. Hu et al conducted a meta-analysis of three randomized clinical trials that included 509 female patients to study the correlation between the size of the endotracheal tubes and the development of sore throat. Small size tubes $(6 \mathrm{~mm})$ were associated with a significant reduction in POST and in obtaining better postoperative clinical outcomes. ${ }^{23}$ Additionally, Biro et al proposed that the use of large endotracheal tubes (size $=7.5 \mathrm{~mm}$ ) may have been the factor behind the increased incidence of sore throat. $^{2}$ On the other hand, no significant difference was noticed between male and female patients in the incidence of POST following endotracheal intubation although larger tubes $($ size $=6.0 \mathrm{~mm}$ ) were used with females than these with male patients $($ size $=8.0 \mathrm{~mm})(\mathrm{p}=0.20) .{ }^{24}$ However, lower incidence rates were noticed among the female $(27 \%)$ versus male patients $(38 \%)$. Therefore, a reduction in the tube size, not only the internal diameter, should be approached to decrease the incidence of POST and other related symptoms and complications that patients may experience.

\section{POST and endotracheal tube types}

In addition to the size and diameters of the tubes, the type has been also reported to be an associated factor. For instance, Maruyama et al and Najafi et al results showed that using the airway scope (Pentax, Tokyo, Japan), and the GlideScope $^{\circledR}$ (Verathon Inc., Bothell, WA, USA) were significantly associated with reduced incidence rates than using the direct laryngoscopy. ${ }^{25,26}$ Moreover, both studies have used relatively big tubes with an underestimation of the tracheal cuff pressures which may have influenced the results. One more risk factor is the use of double-lumen tubes as reported by Clayton-Smith while single-lumen tubes appear to have no effect. ${ }^{27,28}$ Moreover, using bronchial blockers and intubation while the tracheal lumen is positioned anteriorly during insertion through the vocal cords were both associated with lower rates. ${ }^{27,29,30}$

\section{Prevention against POST following endotracheal intubation}

\section{Steroid therapy}

Among the steroid family, most studies seem to use preoperative Dexamethasone only for the prevention of the development of POST after endotracheal intubation. Kuriyama et al meta-analysis of 20 RCTs concluded that topical administration of corticosteroids was significantly associated with reduced rates of POST following tracheal intubation. ${ }^{31}$ Moreover, Sun et al performed a metaanalysis of seven randomized controlled trials (RCTs) that studied the effect of intravenous corticosteroid administration reduced POST following endotracheal intubation. ${ }^{32}$ The results showed that IV corticosteroids significantly reduced the severity and rate of POST 24 hours following the extubation. However, it is worth mentioning that the smallest intubated tube size was 7.0 $\mathrm{mm}$, and the optimal effective dose was adjusted to 0.1 $\mathrm{mg} / \mathrm{kg}$. Moreover, topical triamcinolone was also effective in reducing the rate and severity of POST 24 hours after application more than chlorhexidine. ${ }^{33}$ Similarly, inhaled budesonide and fluticasone were also effective in reducing POST. ${ }^{34}$ A previous study also proved that topical betamethasone was better than $2 \%$ lidocaine gel in reducing the incidence and severity. ${ }^{35}$ Singh et al conducted a meta-analysis of 42 trials comparing eight treatment regimens and found that inhaled corticosteroid therapy was the probably the best treatment regimen to reduce the early development of sore throat (4-6 hours from extubation). ${ }^{36}$

\section{Licorice therapy}

It is a compound derived from the roots of the Glycyrrhiza glabra plant and in being extracted and used in the modern and traditional medicine due to its proven efficacy as an anti-inflammatory and anti-tussive agent that can lead to the prevention of POST. In 2018, Kuriyama et al conducted a meta-analysis of five RCTs involving 609 patients and found that topical administration of licorice has significantly reduced the incidence and severity of POST following tracheal intubation. ${ }^{37}$ Moreover, no side effects were estimated among the studies that investigated this regimen. In the same context, Agarwal et al demonstrated that pre-anesthetic licorice gargling was significantly associated with reduced early POST, however, no difference was noticed 24 hours after the operation between the licorice and placebo groups. ${ }^{38}$ Similarly, Ruetzler et al showed similar results, however, the authors used double-lumen endotracheal tubes. Preoperative suction of licorice lozenges was also reported as an effective measurement for POST prevention. ${ }^{39,40}$

\section{NSAIDs}

These compounds have been widely used in many disorders as prophylactic measurements in addition to its therapeutic anti-inflammatory effect on many diseases. In the prevention of POST, benzydamine hydrochloride has been the main NSAID agent that is widely reported in published trials. Kuriyama et al conducted a meta-analysis to study the efficacy of topical benzydamine hydrochloride and its effect on POST following endotracheal intubation. ${ }^{41} \mathrm{~A}$ total of $30 \mathrm{RCTs}$ were included and these recruited a total of 1842 patients. The results showed that topical benzydamine hydrochloride was significantly associated with reduced incidence rates of POST, however, it was not significant in reducing the severity. The 
application of benzydamine hydrochloride has been reported to be used as a spray over the tracheal tube or the oropharynx as reported by some studies while others preferred to use it as a gargled form. An older metaanalysis of eight studies concluded that topical benzydamine hydrochloride effectively reduced the severity and incidence of POST following tracheal intubation at all periodic intervals following the operations. ${ }^{42}$ The administration of aspirin and diclofenac has been also reported in the prevention of POST. Aspirin was found to reduce the incidence and severity of POST, however, it lasted for two hours only. ${ }^{43}$ On the other hand, the administration of IV diclofenac showed no improved outcomes, however, when used topically it decreased the incidence. ${ }^{44,45}$

\section{Lidocaine therapy}

Lidocaine therapy has been validated in the prevention of POST following endotracheal intubation by the largest made and relative meta-analysis by the Cochrane collaboration where they have analyzed the results of 19 RCTs involving the different ways of lidocaine administration in 1940 patients for prevention of POST. ${ }^{46}$ Lidocaine was applied inside the tracheal tube, over the tube, IV, and as an aerosol spray in the different included studies. Although the results showed that IV and spray regimens were both associated with reduced incidence rates, no significance was found when high-quality studies were the only studies that were included in the analysis which reflects the effect of poor-quality studies in influencing the results and causing bias. Additionally, Mekhemar et al and Kuriyama et al results showed that benzydamine hydrochloride application significantly reduced the incidence and severity of POST when compared to topical administration of both $10 \%$ and $5 \%$ lidocaine. ${ }^{41,47}$ Besides, Mekhemar's study even found that increased incidence rates were associated with the $10 \%$ lidocaine group when compared to the normal saline one. ${ }^{47}$ Therefore, lidocaine should not be approached for the prevention of POST following endotracheal intubation.

\section{Other preventive modalities}

N-methyl-D-aspartate (NMDA) receptor antagonists have been also reported to reduce the incidence and severity of POST. Topical magnesium and ketamine administration have been widely reported among various clinical trials. Singh et al conducted a meta-analysis of seven RCTs involving 726 patients where topical magnesium was applied as a prophylactic measurement against POST following tracheal intubation. ${ }^{48}$ The results showed that the incidence of POST was significantly reduced in these patients compared to other control groups. Similar results were reported by Kuriyama et al which indicated the significance of magnesium in reducing the incidence and severity of POST. ${ }^{49}$ Ketamine administration, on the other hand, was investigated by a large meta-analysis of 41 RCTs involving 3784 patients. ${ }^{50}$ The results showed that topical ketamine administration was significantly associated with reduced incidence rates and severity of POST following postoperative intubation. Additionally, the analysis showed that topical administration was less efficacious than the combined use of nebulized ketamine and clonidine.

Other preventive measures that may reduce the incidence and severity of POST following endotracheal intubation include the continuous and careful monitoring of the tracheal tube cuff pressure as reported by previous controlled studies which showed that using a manometer, for instance, was significantly associated with decreased incidence and severity. ${ }^{51-54}$ Moreover, many recent modalities and regimens have been approached as lozenges (amylmetacresol), dexpanthenol, gabapentin, and azulene. ${ }^{55-59}$ However, these studies were reported by trials of limited numbers of patients only, and gabapentin efficacy in preventing sore throat was not present during swallowing and was only limited to preventing it at rest. ${ }^{59}$ Therefore, future studies with bigger sample sizes are recommended for further validation of these modalities.

\section{CONCLUSION}

POST following endotracheal intubation remains an annoying side effect in patients following general anesthesia. The prevention of this phenomenon is basically dependant on the application of some procedures involving both prophylactic and therapeutic approaches. As a first step for decreasing the incidence, the underlying risk factors should be carefully accounted for. The most common risk factors include ages less than 60 years of age, female sex, cough, asthma, underlying chest diseases, type and timing of the operation, type, and size of the used endotracheal tube. Moreover, the experience of the personnel intubating the patients may have been another risk factor. Medical prevention of POST can be achieved by several agents as steroids, NSAIDS, NMDA receptor antagonists, careful monitoring of the tracheal cuff pressure, and licorice application. On the other hand, using lidocaine has been contraindicated according to previous meta-analysis results. Additionally, we recommend that future studies with bigger sample sizes are should be conducted for further validation of the new modalities.

Funding: No funding sources

Conflict of interest: None declared

Ethical approval: Not required

\section{REFERENCES}

1. Macario A, Weinger M, Truong P, Lee M. Which Clinical Anesthesia Outcomes Are Both Common and Important to Avoid? The Perspective of a Panel of Expert Anesthesiologists. Anesth Analgesia. 1999;88(5).

2. Biro P, Seifert B, Pasch T. Complaints of sore throat after tracheal intubation: a prospective evaluation. Eur J Anaesthesiol. 2005;22(4):307-11. 
3. Cho C-K, Kim J-E, Yang H-J, Sung T-Y, Kwon H, Kang P-S. The effect of combining lidocaine with dexamethasone for attenuating postoperative sore throat, cough, and hoarseness. Anesthesia Pain Med. 2016;11:42-8.

4. Higgins PP, Chung F, Mezei G. Postoperative sore throat after ambulatory surgery. Bri J Anaesth. 2002;88(4):582-4.

5. Scuderi PE. Postoperative Sore Throat: More Answers Than Questions. Anesth Analgesia. 2010;111(4).

6. Coelho R, Paiva T, Mathias L. In vitro evaluation of the method effectiveness to limit inflation pressure cuffs of endotracheal tubes. Revista Brasileira de Anestesiologia. 2016;66.

7. Hu B, Bao R, Wang X. The Size of Endotracheal Tube and Sore Throat after Surgery: A Systematic Review and Meta-Analysis. PloS one. 2013;8:74467.

8. Al-Qahtani A, Messahel F. Quality improvement in anesthetic practice - Incidence of sore throat after using small tracheal tube. Middle East J Anesthesiol. 2005;18:179-83.

9. Von Ungern-Sternberg BS, Ramgolam A, Hall GL, Sly PD, Habre W. Peri-operative adverse respiratory events in children. Anaesthesia. 2015;70(4):440-4.

10. Minamiguchi M, Tanaka Y, Kitagawa K, Inoue S, Kawaguchi M. Evaluation of factors associated with postoperative sore throat. Masui Japanese J Anesthesiol. 2014;63:401-5.

11. Stout DM, Bishop MJ, Dwersteg JF, Cullen BF. Correlation of endotracheal tube size with sore throat and hoarseness following general anesthesia. Anesthesiology. 1987;67(3):419-21.

12. Jaensson M, Olowsson L, Nilsson U. Endotracheal tube size and sore throat following surgery: a randomized-controlled study. Acta Anaesthesiologica Scandinavica. 2010;54(2):147-53.

13. Jung H, Kim H. Dexamethasone contributes to the patient management after ambulatory laryngeal microsurgery by reducing sore throat. Eur Arch Otorhino-laryngol. 2013;270.

14. Hung N-K, Wu C-T, Chan S-M. Effect on Postoperative Sore Throat of Spraying the Endotracheal Tube Cuff with Benzydamine Hydrochloride, 10\% Lidocaine, and 2\% Lidocaine. Anesth Analgesia. 2010;111(4):882-6.

15. Agarwal A, Nath SS, Goswami D, Gupta D, Dhiraaj S, Singh PK. An Evaluation of the Efficacy of Aspirin and Benzydamine Hydrochloride Gargle for Attenuating Postoperative Sore Throat: A Prospective, Randomized, Single-Blind Study. Anesth Analgesia. 2006;103(4):1001-3.

16. Maruyama K, Yamada T, Hara K. Effect of clonidine premedication on postoperative sore throat and hoarseness after total intravenous anesthesia. J Anesth. 2006;20:327-30.

17. Ahuja V, Mitra S, Sarna R. Nebulized ketamine decreases incidence and severity of post-operative sore throat. Indian J Anaesth. 2015;59(1):37-42.
18. Lee JY, Sim WS, Kim ES. Incidence and risk factors of postoperative sore throat after endotracheal intubation in Korean patients. J Int Med Res. 2017;45(2):744-52.

19. Inoue S, Abe R, Tanaka Y, Kawaguchi M. Tracheal intubation by trainees does not alter the incidence or duration of postoperative sore throat and hoarseness: a teaching hospital-based propensity score analysis $\dagger$. Br J Anaesth. 2015;115(3):463-9.

20. Jaensson M, Gupta A, Nilsson U. Risk factors for development of postoperative sore throat and hoarseness after endotracheal intubation in women: A secondary analysis. AANA J. 2012;80:67-73.

21. Myles PS, Hunt JO, Moloney JT. Postoperative 'minor' complications. Anaesthesia. 1997;52(4):3006.

22. Jaensson M, Gupta A, Nilsson UG. Gender differences in risk factors for airway symptoms following tracheal intubation. Acta Anaesthesiologica Scandinavica. 2012;56(10):130613.

23. Hu B, Bao R, Wang X. The size of endotracheal tube and sore throat after surgery: a systematic review and meta-analysis. PLoS One. 2013;8(10):74467.

24. Jaensson M, Gupta A, Nilsson U. Gender differences in sore throat and hoarseness following endotracheal tube or laryngeal mask airway: a prospective study. BMC Anesthesiol. 2014;14(1):56.

25. Maruyama K, Nakagawa H, Imanishi H, Kitamura A, Hayashida M. Comparison of postoperative pharyngeal morbidity using the Macintosh laryngoscope or AirWay Scope after mastectomy. J Anesth. 2011;25(5):773.

26. Najafi A, Imani F, Makarem J. Postoperative sore throat after laryngoscopy with macintosh or glide scope video laryngoscope blade in normal airway patients. Anesth Pain Med. 2014;4(1):15136.

27. Clayton-Smith A, Bennett K, Alston R. A Comparison of the Efficacy and Adverse Effects of Double-Lumen Endobronchial Tubes and Bronchial Blockers in Thoracic Surgery: A Systematic Review and Meta-analysis of Randomized Controlled Trials. J Cardiothorac Vasc Anesth. 2014;29.

28. Turkstra TP, Smitheram AK, Alabdulhadi O, Youssef H, Jones PM. The Flex-Tip ${ }^{\mathrm{TM}}$ tracheal tube does not reduce the incidence of postoperative sore throat: a randomized controlled trial. Canad J Anesth J. 2011;58(12):1090-6.

29. Seo JH, Kwon TK, Jeon Y, Hong DM, Kim HJ, Bahk $\mathrm{JH}$. Comparison of techniques for double-lumen endobronchial intubation: 90\&\#xb0; or 180\&\#xb0; rotation during advancement through the glottis. $\mathrm{Br}$ Jf Anaesth. 2013;111(5):812-7.

30. Zhong T, Wang W, Chen J, Ran L, Story DA. Sore Throat or Hoarse Voice with Bronchial Blockers or Double-Lumen Tubes for Lung Isolation: A Randomised, Prospective Trial. Anaesth Intensive Care. 2008;36(6):441-6.

31. Kuriyama A, Maeda H, Sun R, Aga M. Topical application of corticosteroids to tracheal tubes to 
prevent postoperative sore throat in adults undergoing tracheal intubation: a systematic review and meta-analysis. Anaesthesia. 2018;73(12):154656.

32. Sun L, Guo R. Dexamethasone for preventing postoperative sore throat: A meta-analysis of randomized controlled trials. Irish J Med Sci. $2013 ; 183$.

33. Park SY, Kim SH, Lee SJ. Application of triamcinolone acetonide paste to the endotracheal tube reduces postoperative sore throat: a randomized controlled trial. Canad J Anesth. 2011;58(5):436-42.

34. Tazeh-Kand NF, Eslami B, Mohammadian K. Inhaled fluticasone propionate reduces postoperative sore throat, cough, and hoarseness. Anesth Analg. 2010;111(4):895-8.

35. Sumathi PA, Shenoy T, Ambareesha M, Krishna HM. Controlled comparison between betamethasone gel and lidocaine jelly applied over tracheal tube to reduce postoperative sore throat, cough, and hoarseness of voice. Br J Anaesth. 2007;100(2):2158.

36. Singh NP, Makkar JK, Cappellani RB, Sinha A, Lakshminarasimhachar A, Singh PM. Efficacy of topical agents for prevention of postoperative sore throat after single lumen tracheal intubation: a Bayesian network meta-analysis. Canad J Anesth. 2020;67(11):1624-42.

37. Kuriyama A, Maeda H. Topical application of licorice for prevention of postoperative sore throat in adults: A systematic review and meta-analysis. J Clin Anesth. 2019;54:25-32.

38. Agarwal A, Gupta D, Yadav G, Goyal P, Singh PK, Singh U. An evaluation of the efficacy of licorice gargle for attenuating postoperative sore throat: a prospective, randomized, single-blind study. Anesth Analg. 2009;109(1):77-81.

39. Ruetzler K, Fleck M, Nabecker S. A Randomized, Double-Blind Comparison of Licorice Versus SugarWater Gargle for Prevention of Postoperative Sore Throat and Postextubation Coughing. Anesth Analgesia. 2013;117(3):614-21.

40. Gupta D, Agrawal S, Sharma J. Effect of preoperative licorice lozenges on incidence of postextubation cough and sore throat in smokers undergoing general anesthesia and endotracheal intubation. Middle East J Anesthesiol. 2013;22:173-8.

41. Kuriyama A, Aga M, Maeda H. Topical benzydamine hydrochloride for prevention of postoperative sore throat in adults undergoing tracheal intubation for elective surgery: a systematic review and metaanalysis. Anaesthesia. 2018;73(7):889-900.

42. Chen C-y, Kuo C-J, Lee Y-W, Lam F, Tam K-W. Benzydamine hydrochloride on postoperative sore throat: A meta-analysis of randomized controlled trials. Canad J Anaesth. 2013;61.

43. An Evaluation of the Efficacy of Aspirin and Benzydamine Hydrochloride Gargle for Attenuating Postoperative Sore Throat: A Prospective,
Randomized, Single-Blind Study. Survey Anesthesiol. 2007;51(5):269.

44. Thang'a P, Kamya D, Mung'ayi V. Effects of intravenous diclofenac on postoperative sore throat in patients undergoing laparoscopic surgery at Aga Khan University Hospital, Nairobi: a prospective, randomized, double blind controlled trial. Afr Health Sci. 2013;13(4):999-1006.

45. Rahimi M, Makarem J. Effects of Diclofenac Epolamine Patch on Postoperative Sore Throat in Parturients After Cesarean Delivery Under Endotracheal General Anesthesia. Acta Anaesthesiologica Taiwanica. 2009;47(1):17-21.

46. Tanaka Y, Nakayama T, Nishimori M, Tsujimura Y, Kawaguchi M, Sato Y. Lidocaine for preventing postoperative sore throat. Cochrane Database Systematic Rev. 2015(7).

47. Mekhemar NA, El-agwany AS, Radi WK, El-Hady SM. Comparative study between benzydamine hydrochloride gel, lidocaine $5 \%$ gel and lidocaine $10 \%$ spray on endotracheal tube cuff as regards postoperative sore throat. Braz J Anesthesiol. 2016;66(3):242-8.

48. Singh NP, Makkar JK, Wourms V, Zorrilla-Vaca A, Cappellani RB, Singh PM. Role of topical magnesium in post-operative sore throat: A systematic review and meta-analysis of randomised controlled trials. Indian J Anaesth. 2019;63(7):520-9.

49. Kuriyama A, Maeda H, Sun R. Topical application of magnesium to prevent intubation-related sore throat in adult surgical patients: a systematic review and meta-analysis. Canad J Anesth. 2019;66(9):1082-94.

50. Kuriyama A, Nakanishi M, Kamei J, Sun R, Ninomiya K, Hino M. Topical application of ketamine to prevent postoperative sore throat in adults: A systematic review and meta-analysis. Acta Anaesth Scand. 2020;64(5):579-591.

51. Liu J, Zhang X, Gong W. Correlations Between Controlled Endotracheal Tube Cuff Pressure and Postprocedural Complications: A Multicenter Study. Anesthe Analgesia. 2010;111(5):1133-7.

52. Ansari L, Bohluli B, Mahaseni H, Valaei N, SadrEshkevari P, Rashad A. The effect of endotracheal tube cuff pressure control on postextubation throat pain in orthognathic surgeries: A randomized doubleblind controlled clinical trial. Br J Oral Maxillofacial Surg. 2013;52.

53. Koo C-H, Sohn H-M, Choi E-S. The Effect of Adjustment of Endotracheal Tube Cuff Pressure during Scarless Remote Access Endoscopic and Robotic Thyroidectomy on Laryngo-Pharyngeal Complications: Prospective Randomized and Controlled Trial. J Clin Med. 2019;8(11):1787.

54. Ryu J-H, Han S-S, Do S-H, Lee J-M, Lee S-C, Choi E-S. Effect of Adjusted Cuff Pressure of Endotracheal Tube During Thyroidectomy on Postoperative Airway Complications: Prospective, Randomized, and Controlled Trial. World J Surg. 2013;37(4):786-91. 
55. Ebneshahidi A, Mohseni M. Strepsils $®$ tablets reduce sore throat and hoarseness after tracheal intubation. Anesth Analg. 2010;111(4):892-4.

56. Gulhas N, Canpolat H, Cicek M. Dexpanthenol pastille and benzydamine hydrochloride spray for the prevention of post-operative sore throat. Acta anaesth Scand. 2007;51:239-43.

57. Gupta D, Agrawal S, Sharma JP. Evaluation of preoperative Strepsils lozenges on incidence of postextubation cough and sore throat in smokers undergoing anesthesia with endotracheal intubation. Saudi J Anaesth. 2014;8(2):244-8.

58. Ogata J, Minami K, Horishita T. Gargling with Sodium Azulene Sulfonate Reduces the
Postoperative Sore Throat After Intubation of the Trachea. Anesth Analgesia. 2005;101(1):290-3.

59. Cook T, Howes B. Supraglottic airway devices: recent advances. Cont Educ Anaesth Crit Care Pain. 2010;11(2):56-61.

Cite this article as: El Sharkawy AM, Rawas MM, Altoies AM, Al Arbash MA, Alzahrani AA, Alnahari AM, et al. Management of post-operative sore throat in patients undergoing endotracheal intubation: literature review. Int J Community Med Public Health 2021;8:855-61. 